\title{
Namoro na adolescência no Brasil: circularidade da violência psicológica nos diferentes contextos relacionais
}

\author{
Adolescent dating in Brazil: the circularity \\ of psychological violence in different relationship contexts
}

Queiti Batista Moreira Oliveira ${ }^{1}$

Simone Gonçalves de Assis ${ }^{1}$

Kathie Njaine ${ }^{1}$

Thiago Oliveira Pires ${ }^{1}$

${ }^{1}$ Centro Latino-Americano de Estudos de Violência e Saúde Jorge Careli, Escola Nacional de Saúde Pública Sergio Arouca, Fundação Oswaldo Cruz. Av. Brasil 4036/700, Manguinhos.

21.040-361 Rio de Janeiro RJ Brasil.

queitioliveira@ig.com.br
Abstract The scope of this paper is to evaluate the perpetration of psychological violence in current male and female dating relationships and their link to psychological violence experienced in other contexts of their lives, namely family, relationships with friends and dating partners. 3,205 students in the 2nd year of high school (15 to 19 years old) in public and private schools in ten Brazilian cities filled out a closed and selfadministered questionnaire. The results highlight the fact that the increase in the number of psychologically violent events perpetrated by adolescents in their intimate relationships is related to greater verbal aggression of the mother and father, and the more frequent experiences of psychological violence between parents, siblings, friends and that existing in earlier dating relationships. This reinforces the notion of circularity of psychological violence in various contexts of socialization of adolescents and highlights the continuity of aggressive behavior in other dating relationships, and those between siblings, family and friends.

Key words Psychological violence, Dating violence, Adolescence, Family, Friends, Peers
Resumo Avalia a perpetração de violência psicológica no relacionamento afetivo-sexual atual de adolescentes do sexo masculino e feminino e sua relação com violência psicológica vivenciada em outros contextos de suas vidas: família, relacionamento com amigos e com parceiros afetivo-sexuais anteriores. Responderam a um questionário fechado e autoaplicado 3.205 escolares do $2^{\circ}$ ano do Ensino Médio, com idade entre 15 e 19 anos, de escolas públicas estaduais e particulares das capitais de dez estados brasileiros. Os resultados destacam que o aumento do número de eventos de violência psicológica perpetrada pelos adolescentes em seus relacionamentos íntimos está relacionado à mais elevada agressão verbal da mãe e do pai; e à mais frequente vivência de violência psicológica entre pais, irmãos, amigos e àquela presente nos namoros anteriores. Reforçam a noção de circularidade da violência psicológica nos diversos contextos de socialização do adolescente e destacam a continuidade do comportamento agressivo em outras relações de namoro, entre irmãos, na família e amigos.

Palavras-chave Violência psicológica, Violência no namoro, Adolescentes, Família, Amigos, Pares 


\section{Introdução}

A violência entre namorados adolescentes é considerada um problema de saúde pública na medida em que suas expressões podem se constituir em padrões de resolução de conflitos que se perpetuam nos relacionamentos íntimos na vida adulta ${ }^{1,2}$. Por esse motivo, as ações de prevenção à violência entre parceiros íntimos precisa se voltar para essa faixa etária, quando as primeiras vivências de relacionamentos afetivo-sexuais estão acontecendo, reproduzindo, mas também transformando normas de gênero que justificam a violência contra o parceiro ${ }^{3,4}$.

A literatura sobre violência entre namorados adolescentes mostra predominância de estudos sobre violência física e sexual, talvez por produzirem efeitos mais visíveis. A violência psicológica ainda tem pouco destaque, embora diversas pesquisas mostrem prevalências de violência psicológica mais altas do que as que têm merecido mais destaque $e^{3,5-9}$. Talvez essa escassez se deva ao fato de que a violência psicológica somente se torne visível a partir de seus efeitos, ou seja, quando se expressa em comportamentos apresentados pelas vítimas ao longo do tempo ${ }^{10}$.

A partir da década de 80 verifica-se o crescente interesse pelo tema da violência psicológica entre namorados adolescentes, com relatos de pesquisas norte-americanas e canadenses ${ }^{11-14}$, portuguesa $^{15}$, espanholas ${ }^{6,16}$, chinesa ${ }^{17}$, suíça ${ }^{18}$, israelenses $^{19}$, mexicanas ${ }^{20,21}$ e brasileiras ${ }^{5,22}$.

Ocorre violência psicológica sobre crianças e adolescentes quando a comunicação existente expõe uma mensagem cultural específica de rejeição ou quando prejudica o processo de socialização e o desenvolvimento psicológico. Dentre os comportamentos mais comuns desta forma de violência tem-se o ato de rejeitar, isolar, aterrorizar, ignorar e corromper ${ }^{23}$. Jezl et al. ${ }^{9}$ consideram que as táticas indiretas de controle do outro e as agressões verbais ou emocionais alcançam um patamar privilegiado nas relações de namoro entre adolescentes.

Nesse cenário de pouca atenção à violência psicológica, Sears et al. ${ }^{3}$ afirmam que o envolvimento de adolescentes em violência no namoro acaba subestimado, pois além de seus altos índices de prevalência, a violência psicológica é a forma mais corriqueiramente perpetrada pelos adolescentes contra seus parceiros, além de ser um preditor da violência física no namoro ${ }^{24,25}$.

Os altos índices de agressões verbais observados entre namorados permitem vislumbrar como esse tipo de violência pode estar sendo ba- nalizada e se conformando como uma forma de comunicação entre os adolescentes em seus relacionamentos afetivo-sexuais. Em um estudo quanti-qualitativo com adolescentes brasileiros, $85,3 \%$ já havia praticado algum tipo de violência verbal contra um parceiro (provocar ciúmes e raiva, depreciar, insultar, falar em tom hostil, dentre outros) e várias manifestações de agressões verbais eram justificadas ou mesmo consideradas aceitáveis pelos adolescentes ${ }^{26}$. Altas prevalências também foram encontradas entre adolescentes espanhóis - 96,3\% ${ }^{6}$. Entre os canadenses, 'falar com o parceiro em tom hostil' foi o item mais relatado de violência psicológica $53,7 \%$, seguido por 'falar sobre algo ruim que o parceiro tinha feito no passado' - 52,65 e por 'provocar ciúmes' - 51.85 27,28 .

Outro aspecto que justifica a relevância de aprofundarmos o conhecimento sobre a violência psicológica no âmbito dos relacionamentos íntimos tanto entre adolescentes quanto entre adultos é sua coocorrência com outros tipos de violência, conforme mostram alguns estudos ${ }^{3,26}$. Entre adolescentes brasileiros, por exemplo, 32,3\% relataram vivenciar em conjunto, violência psicológica e sexual, e 24,9\% convivem simultaneamente com violência psicológica, física e sexual ${ }^{26}$.

Dentre os diferentes fatores elencados por Fernet ${ }^{28}$ como importantes para o desencadeamento da violência entre parceiros adolescentes estão o histórico familiar e experiências de vitimização, as experiências amorosas e sexuais e o meio social. Nesse sentido, diversos estudos relacionam as agressões sofridas e/ou praticadas no namoro a outras vivenciadas ou testemunhadas na família, entre pares e grupos de amigos. Entretanto, novamente nota-se pouco destaque à violência psicológica, pois tais estudos privilegiam a violência física ${ }^{29}$ ou analisam a violência psicológica em conjunto com outros tipos de violência ${ }^{30-35}$, impossibilitando uma reflexão mais focada na violência psicológica em suas especificidades. Kinsfogel e Grych ${ }^{36}$ embora não enfoquem exclusivamente a violência psicológica, concluem que a agressão percebida entre pares, esteve associada aos relatos de conflito e de agressões no namoro, tanto as físicas quanto as verbais/emocionais.

Tendo em mente a possível existência de violência psicológica nos diferentes âmbitos relacionais de adolescentes, o presente artigo tem como objetivo avaliar a perpetração de violência psicológica no relacionamento afetivo-sexual atual de adolescentes do sexo masculino e feminino brasileiros e sua relação com violência psicológica 
vivenciada em outros contextos de suas vidas: família, relacionamento com amigos e com parceiros afetivo-sexuais anteriores.

\section{Metodologia}

\section{Amostra}

O presente estudo utiliza dados de uma pesquisa realizada entre 2007 e 2009 que investigou a prevalência da violência no namoro entre adolescentes entre 15-19 anos de idade ${ }^{5}$. Foi realizado um inquérito epidemiológico com amostra representativa de adolescentes estudantes do $2^{\circ}$ ano do Ensino Médio das escolas públicas estaduais e particulares das capitais de dez estados brasileiros totalizando 3.205 jovens. Esse tipo de estudo adotado permite aferir associação entre variáveis, e não causalidades. As cidades foram selecionadas por conveniência dentre aquelas com mais altas taxas de violência entre jovens, no ano de 2007. São alunos entre 15 e 19 anos de idade e que já namoraram ou "ficaram" com algum parceiro afetivo-sexual.

O plano amostral do projeto foi composto por vinte estratos, em função das dez capitais brasileiras, sendo duas em cada região do país: Manaus/AM, Porto Velho/RO, Recife/PE, Teresina/PI, Brasília/DF, Cuiabá/MT, Rio de Janeiro/ RJ, Belo Horizonte/MG, Florianópolis/SC e Porto Alegre/RS; e da natureza da instituição de ensino (pública e particular). A amostragem conglomerada multi-estágio, com seleção em duas etapas: $1^{\text {a }}$ etapa - escolha das escolas, com probabilidade de seleção proporcional à quantidade de alunos (PPT sistemática) de $2^{\circ}$ ano em cada um dos vinte estratos; $2^{\text {a }}$ etapa - uma turma foi selecionada aleatoriamente, dentro da escola, para a aplicação do questionário com todos os alunos.

\section{Variáveis}

A variável dependente utilizada é a escala Conflict in Adolescent Dating Relationships Inventory - $C A D R I^{28}$, que avalia a violência psicológica sofrida e perpetrada nos relacionamentos afetivossexuais (namoro ou "ficar") entre adolescentes. Neste artigo apresentam-se apenas dados da violência psicológica perpetrada pelo adolescente, diferenciada segundo os tipos: a) verbal/emocional, com itens tais como mencionar algo de ruim que o parceiro fez no passado, dizer coisas para deixá-lo com raiva, falar com ele em tom de voz hostil, insultar, ridicularizar na frente dos outros
(10 itens); b) relacional: tentar virar os amigos contra o parceiro, dizer coisas sobre ele aos seus amigos, para virá-los contra ele, espalhar boatos sobre ele (3 itens); c) ameaças: destruir ou ameaçar destruir algo de valor do parceiro, tentar amedrontá-lo, ameaçar machucá-lo, ameaçar bater nele ou jogar coisa sobre ele (4 itens). Os resultados apresentados neste artigo agregam as três subescalas, considerando a totalidade de atos de violência psicológica perpetrada. As opções de resposta relativas aos últimos 12 meses são: nunca, raramente (aconteceu 1 ou 2 vezes), às vezes ( 3 a 5 vezes) e sempre ( 6 vezes ou mais). Cada opção de resposta foi recategorizada neste artigo da seguinte forma: nunca $=0$; raramente $=1,5$ vezes, que representa a média da frequência assinalada; às vezes $=4$ (média da frequência assinalada); sempre $=6$ (limite inferior da frequência assinalada, buscando não inflacionar os resultados). As pontuações de cada um desses itens foram somadas e seus escores foram computados (arredondados para números inteiros no modelo), visando avaliar a frequência/continuidade dos atos de violência psicológica perpetrada no namoro/ficar. A soma possível dos escores permite valores entre 0 e 102 pontos derivados dos 17 itens investigados. A escala foi adaptada transculturalmente para a língua portuguesa ${ }^{5}$.

Como variáveis independentes tem-se sexo e idade (15 a 19 anos), bem como as seguintes questões/escalas de violência psicológica:

Conflict Tactics Scale ${ }^{37}$, que afere a violência familiar dos pais contra os filhos. Consiste de uma lista de ações que pode tomar um membro da família quando em conflito com outro. Neste artigo foi utilizada a subescala com 6 itens que avalia a agressão verbal praticada pelo pai e pela mãe sobre o(a) adolescente entrevistado(a) (últimos 12 meses), a partir de atos como xingar ou insultar, ficar emburrado, chorar, fazer coisas para irritar, ameaçar bater ou jogar coisas sobre o adolescente, destruir, bater ou chutar objetos. A escala foi validada para a população brasilei$\mathrm{ra}^{38}$. As opções de respostas foram codificadas em 0 -nunca, 1-algumas vezes e 2 -muitas vezes, permitindo a obtenção de escores individuais entre 0 e 12 pontos. Escores foram construídos com a soma desses itens buscando avaliar a continuidade dos atos de violência psicológica dos responsáveis sobre o adolescente.

Outras formas de violência psicológica foram indagadas através de perguntas isoladas, que se referem ao tipo de relação existente, sem mensuração de tempo: se os pais humilham um ao outro (presença/ausência); se ocorreu agressão 
verbal em relacionamentos afetivo-sexuais anteriores do adolescente (presença/ausência); se há resolução de conflitos entre amigos através de xingamentos e humilhações (presença/ausência); e se há brigas entre irmãos envolvendo xingamentos e humilhações.

\section{Análise}

Foram aplicados modelos de regressão binomial negativa inflacionada de zeros, por assumir que a variável resposta é uma contagem (frequência de violência psicológica perpetrada) com uma alta quantidade de zeros (respostas negativas) e devido a sua variância ser muito maior do

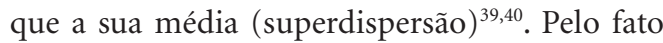
dos dados modelados serem originados de amostragem complexa, informações sobre o plano amostral foram inseridas para correção das medidas de precisão e pesos para correção das medidas pontuais.

Partiu-se inicialmente de um modelo completo (que agregava todas as variáveis independentes) para explicar a frequência de violência psicológica perpetrada no namoro ou no "ficar". Cada tipo de violência foi analisada separadamente, totalizando seis modelos múltiplos ajustados (agressão verbal materna e paterna, violência entre pais, irmãos, amigos e em relacionamentos afetivo-sexuais anteriores), avaliando-se em cada um a presença de interação dessas variáveis com sexo e idade, retirando as variáveis não estatisticamente significativas ao nível de 0,05.

$\mathrm{Na}$ pesquisa original ${ }^{5}$ adolescentes foram questionados a respeito de outros tipos de violência (física e sexual), outras formas relacionais quais como violência entre irmãos, violência intrafamiliar, entretanto, no presente estudo, a metodologia adotada não se propôs a controlar a violência psicológica em relação às demais formas de violência. $\mathrm{O}$ pacote estatístico utilizado foi o Stata versão 10 .

\section{Resultados}

Como se pode observar na Tabela 1 , há maior presença de mulheres $(62,6 \%)$ e de jovens da idade de 16 anos (54,1\%) na amostra investigada. No que se refere a convivência corriqueira com a violência em outros contextos, tem-se que a violência entre irmãos é a mais frequente (75,6\%), seguida pela que se dá entre amigos $(46,3 \%)$ e a que ocorreu em relacionamentos afetivo-sexuais anteriores do adolescente $(29,8 \%)$. O relato da existência de violência psicológica entre os pais foi mencionada por $25 \%$ dos entrevistados. No que tange a agressão verbal do pai sobre os 3.205 adolescentes investigados neste artigo, encontrou-se a média de 2,1 pontos positivos no escore - dentre os 6 itens que compõem a escala. Frequência um pouco maior foi encontrada em relação à agressão verbal materna (média de 3,2 pontos).

Quanto a variável dependente violência psicológica perpetrada no namoro pelo adolescente, encontrou-se a média de 14,1 pontos positivos entre os 3.205 estudantes investigados, considerando-se o somatório das respostas das 17 questões que aferem este tipo de violência.

O próximo passo foi efetuar a modelagem dos dados, analisando sexo e idade em um modelo simples. Como a variável idade não foi estatisticamente significativa ( $\mathrm{p}=0,344)$, foi excluída das análises seguintes.

Na Tabela 2 estão apresentados os resultados para cada um dos seis modelos, que se referem aos âmbitos vivenciados pelos adolescentes: familiar, com amigos e em relacionamentos afetivo-sexuais anteriores analisados de forma independente.

A seguir analisam-se os resultados obtidos na parte não inflacionada dos modelos, de interesse para o presente artigo.

No primeiro modelo avalia-se a violência psicológica perpetrada pelo adolescente no namoro ou no "ficar" em relação à agressão verbal materna e ao sexo do adolescente. Verifica-se a presença mais frequente de perpetração de violência no namoro ou "ficar" pelas mulheres $(18,4 \%$ exponencial do coeficiente $=1,184)$ quando comparadas aos homens, na presença de agressão verbal da mãe (modelo 1). Observa-se ainda que esta tendência feminina de maior perpetração de violência no namoro é constante para os demais contextos relacionais investigados: a) 21,5\% a mais entre mulheres que sofrem agressão verbal paterna (modelo 2); b) 22\% nas que testemunham a violência psicológica entre pais (modelo 3); c) $21,8 \%$ nas que convivem com violência psicológica entre irmãos (modelo 4); d) 27,8\% dentre as com relacionamentos afetivo-sexuais anteriores com violência psicológica (modelo 5); e) $26,6 \%$ entre as que vivenciam violência psicológica entre amigos (modelo 6).

No que se refere ao número de eventos de violência psicológica perpetrado por adolescentes no namoro ou "ficar" tem-se um incremento de 5,5\% (exponencial do coeficiente $=1,055$ ) a cada aumento de uma unidade no escore de agressão verbal da mãe, levando-se em consideração 
Tabela 1. Descrição das variáveis de análise.

\begin{tabular}{|c|c|c|}
\hline Variáveis & $\mathbf{N}$ & $\%$ \\
\hline \multicolumn{3}{|l|}{ Sexo do adolescente } \\
\hline Feminino & 1897 & 62,6 \\
\hline Masculino & 1305 & 37,4 \\
\hline \multicolumn{3}{|l|}{ Idade do adolescente } \\
\hline 15 & 473 & 10,8 \\
\hline 16 & 1705 & 54,1 \\
\hline 17 & 725 & 23,1 \\
\hline 18 & 231 & 10,3 \\
\hline 19 & 71 & 1,8 \\
\hline \multicolumn{3}{|l|}{ Violência psicológica entre pais } \\
\hline Presença & 760 & 25,0 \\
\hline Ausência & 2324 & 75,0 \\
\hline \multicolumn{3}{|l|}{ Violência psicológica entre amigos } \\
\hline Presença & 1706 & 46,3 \\
\hline Ausência & 1441 & 53,7 \\
\hline \multicolumn{3}{|l|}{ Violência psicológica entre irmãos } \\
\hline Presença & 2226 & 75,6 \\
\hline Ausência & 541 & 24,4 \\
\hline \multicolumn{3}{|c|}{ Violência psicológica em namoros passados do adolescente } \\
\hline Sim & 971 & 29,8 \\
\hline \multirow[t]{2}{*}{ Não } & 2198 & 70,2 \\
\hline & Média (DP) & Mediana (DIQ) \\
\hline \multicolumn{3}{|c|}{ Violência psicológica perpetrada no namoro pelo adolescente } \\
\hline Escore & $14,1 \quad(12,9)$ & $12(16)$ \\
\hline \multicolumn{3}{|c|}{ Agressão verbal da mãe sobre o adolescente } \\
\hline Escore & $3,2 \quad(2,8)$ & $(4)$ \\
\hline \multicolumn{3}{|c|}{ Agressão verbal do pai sobre o adolescente } \\
\hline Escore & $2,1 \quad(2,5)$ & $1 \quad(3)$ \\
\hline
\end{tabular}

DP: Desvio-padrão; DIQ: Distância interquartílica.

o sexo do adolescente (modelo 1). Este resultado de continuidade do incremento de violência psicológica perpetrada no namoro também se mostra como uma tendência: a) eleva-se 5,5\% à medida que aumenta uma unidade no escore de agressão verbal do pai (modelo 2); b) cresce 18,1\% com a presença do testemunho de violência psicológica entre os pais (modelo 3); c) aumenta $18,6 \%$ com a presença de conflitos entre amigos (modelo 6); d) eleva-se 27,9\% paralelo a presença da violência psicológica entre irmãos (modelo 4); e) eleva-se 53,1\% na presença de violência psicológica em relacionamentos afetivo-sexuais anteriores (modelo 5).

Na parte inflacionada dos modelos observase o aumento de pontos no escore ou a presença de agressões/violências (variáveis independentes) implica em menor chance de pertencer ao grupo que não pratica violência psicológica nas suas relações afetivo-sexuais.

\section{Discussão}

Nossos resultados destacam que o aumento do número de eventos de violência psicológica perpetrada por adolescentes (de ambos os sexos) em seus relacionamentos íntimos está relacionado: a) a mais elevada agressão verbal da mãe e do pai; e b) a mais frequente vivência de violência psicológica entre pais, irmãos, amigos e àquela presente nos namoros anteriores. Denota-se nesses ambientes uma cultura relacional violenta.

A presença de violência psicológica em relacionamentos pregressos acarreta aumento de 53,1\% na ocorrência dessa mesma forma de agressão no relacionamento atual. Em seguida vem a violência entre irmãos, que, quando presente, eleva em 27,9\% essa mesma forma de violência no namoro. Realidade similar ocorre para a violência psicológica entre amigos e entre os pais, que se associam à elevação de 18,6\% e 18,1\%, respectivamente, à violência psicológica no namoro atual. 
Tabela 2. Variáveis associadas com violência psicológica perpetrada nas relações afetivo-sexuais de adolescentes.

\begin{tabular}{|c|c|c|c|c|}
\hline Variáveis & Coef. ${ }^{a}$ & $\mathbf{E P}^{\mathrm{b}}$ & $\operatorname{Exp}(\text { Coef. })^{c}$ & P-valor* \\
\hline \multicolumn{5}{|l|}{ Modelo 1 - Agressão verbal materna e sexo $(\mathrm{N}=2894)$} \\
\hline \multicolumn{5}{|l|}{ Parte não inflacionada } \\
\hline Intercepto & 2,481 & 0,046 & 11,953 & 0,000 \\
\hline Sexo (feminino) & 0,169 & 0,070 & 1,184 & 0,018 \\
\hline Agressão verbal da mãe (escore) & 0,054 & 0,011 & 1,055 & 0,000 \\
\hline \multicolumn{5}{|l|}{ Parte inflacionada } \\
\hline Intercepto & $-1,454$ & 0,103 & 0,234 & 0,000 \\
\hline Agressão verbal da mãe & $-0,153$ & 0,020 & 0,858 & 0,000 \\
\hline \multicolumn{5}{|l|}{ Modelo 2 - Agressão verbal paterna e sexo $(\mathrm{N}=2593)$} \\
\hline \multicolumn{5}{|l|}{ Parte não inflacionada } \\
\hline Intercepto & 2,539 & 0,036 & 12,667 & 0,000 \\
\hline Sexo (feminino) & 0,195 & 0,065 & 1,215 & 0,003 \\
\hline Agressão verbal do pai (escore) & 0,054 & 0,009 & 1,055 & 0,000 \\
\hline \multicolumn{5}{|l|}{ Parte inflacionada } \\
\hline Intercepto & $-1,342$ & 0,230 & 0,261 & 0,000 \\
\hline Sexo (feminino) & $-0,493$ & 0,174 & 0,611 & 0,006 \\
\hline Agressão verbal do pai & $-0,180$ & 0,090 & 0,835 & 0,048 \\
\hline \multicolumn{5}{|c|}{ Modelo 3 - Violência psicológica entre pais e sexo $(\mathrm{N}=2917)$} \\
\hline \multicolumn{5}{|l|}{ Parte não inflacionada } \\
\hline Intercepto & 2,620 & 0,039 & 13,736 & 0,000 \\
\hline Sexo (feminino) & 0,199 & 0,058 & 1,220 & 0,001 \\
\hline Violência psicológica entre pais (presença) & 0,166 & 0,027 & 1,181 & 0,000 \\
\hline \multicolumn{5}{|l|}{ Parte inflacionada } \\
\hline Intercepto & $-1,766$ & 0,136 & 0,171 & 0,000 \\
\hline Violência psicológica entre pais (presença) & $-0,704$ & 0,275 & 0,495 & 0,012 \\
\hline \multicolumn{5}{|c|}{ Modelo 4 - Violência psicológica entre irmãos e sexo $(\mathrm{N}=2613)$} \\
\hline \multicolumn{5}{|l|}{ Parte não inflacionada } \\
\hline Intercepto & 2,472 & 0,108 & 11,846 & 0,000 \\
\hline Sexo (feminino) & 0,197 & 0,068 & 1,218 & 0,005 \\
\hline Violência psicológica entre irmãos (presença) & 0,246 & 0,122 & 1,279 & 0,046 \\
\hline \multicolumn{5}{|l|}{ Parte inflacionada } \\
\hline Intercepto & $-1,836$ & 0,067 & 0,159 & 0,000 \\
\hline \multicolumn{5}{|c|}{$\begin{array}{l}\text { Modelo } 5 \text { - Agressão verbal em relacionamento afetivo-sexual } \\
\text { anterior e sexo }(\mathrm{N}=2995)\end{array}$} \\
\hline \multicolumn{5}{|l|}{ Parte não inflacionada } \\
\hline Intercepto & 2,474 & 0,046 & 11,870 & 0,000 \\
\hline Sexo (feminino) & 0,245 & 0,056 & 1,278 & 0,000 \\
\hline Agressão verbal em relacionamento anterior (presença) & 0,426 & 0,086 & 1,531 & 0,000 \\
\hline \multicolumn{5}{|l|}{ Parte inflacionada } \\
\hline Intercepto & $-1,300$ & 0,166 & 0,273 & 0,000 \\
\hline Sexo (feminino) & $-0,473$ & 0,191 & 0,623 & 0,015 \\
\hline Agressão verbal em relacionamento anterior (presença) & $-2,484$ & 0,461 & 0,083 & 0,000 \\
\hline $\begin{array}{l}\text { Sexo (feminino }{ }^{*} \text { Agressão verbal em relacionamento } \\
\text { anterior (presença) }\end{array}$ & 1,742 & 0,630 & - & 0,007 \\
\hline \multicolumn{5}{|l|}{ Modelo 6 - Violência psicológica entre amigos e sexo $(\mathrm{N}=2971)$} \\
\hline \multicolumn{5}{|l|}{ Parte não inflacionada } \\
\hline Intercepto & 2,544 & 0,045 & 12,730 & 0,000 \\
\hline Sexo (feminino) & 0,236 & 0,067 & 1,266 & 0,001 \\
\hline Violência psicológica entre amigos (presença) & 0,171 & 0,063 & 1,186 & 0,008 \\
\hline \multicolumn{5}{|l|}{ Parte inflacionada } \\
\hline Intercepto & $-1,634$ & 0,127 & 0,195 & 0,000 \\
\hline Violência psicológica entre amigos (presença) & $-0,558$ & 0,240 & 0,572 & 0,022 \\
\hline
\end{tabular}

${ }^{a}$ Coeficiente do modelo; ${ }^{\mathrm{b}}$ Erro-padrão; ${ }^{\mathrm{c}}$ Exponencial do coeficiente. ${ }^{\star}$ Em negrito associações estatisticamente significativas. 
Esses resultados encontram-se controlados segundo o sexo do adolescente e reforçam a noção de circularidade da violência psicológica nos diversos contextos de socialização do adolescente. Dão destaque para a manutenção do comportamento agressivo nos diversos namoros (noção de continuidade) e entre irmãos, mas também na família e entre amigos tal realidade procede.

No que tange à violência parental sofrida algumas pesquisas têm mostrado que os homens e mulheres que sofrem de violência dos pais são significativamente mais propensos a cometer violência em um relacionamento de namoro ${ }^{8,41-45}$. Wolfe et al. ${ }^{27}$ relataram que, entre meninos e meninas de 15 anos de idade, as experiências de maustratos de pais contra filhos foram positivamente relacionadas com o uso de comportamentos psicologicamente abusivos contra o parceiro.

No presente estudo, observa-se situação relevante em relação à violência psicológica paterna e materna praticada contra os filhos, sobretudo nas meninas, estabelecendo-se uma relação progressiva: um incremento de 5,5\% na frequência de violência psicológica na relação afetivo-sexual no namoro/ficar ao se elevar em uma unidade o escore da agressão verbal da mãe ou do pai, controlado pelo sexo do adolescente. Confirmando estes resultados, Price e Byers apud Sears et al. $^{46}$ mostram que experiências de abuso psicológico entre pais e filhos e testemunho de abuso psicológico entre os pais foram positivamente relacionados com a prática de violência psicológica no namoro.

Vale destacar que a literatura mostra que não só sofrer, mas testemunhar violência interparental também é considerado preditor de perpetração violência no namoro em outros estu$\operatorname{dos}^{32,36,44}$. Nessas situações, Simon e Furman ${ }^{32}$ concluem que as percepções dos adolescentes bem como suas avaliações acerca do conflito interparental estavam relacionadas com a quantidade de conflito no relacionamento romântico e com os estilos de conflitos por eles vivenciados. As avaliações dos adolescentes acerca do conflito entre seus pais (isto é, autocensura, ameaça percebida) moderou muitas das associações entre o conflito interparental e comportamento de conflito com parceiros românticos. Os padrões de efeitos moderados diferiram por sexo ${ }^{32}$.

De Kinsfogel e Grych ${ }^{36}$ e Simon e Furman ${ }^{32}$ destacam a importância dos processos sociocognitivos para melhor compreensão das ligações entre a agressão na família e nas relações de namoro, com destaque para a socialização de gênero. Kinsfogel e Grych ${ }^{36}$ concluem que meninos que testemunharam maiores níveis de conflito interparental agressivo eram mais prováveis de perceber a agressão em uma relação de namoro como justificável, predizendo comportamentos hostis e abusivos em relação a parceiros de namoro. Entre as meninas, por sua vez, não foi encontrada correlação entre sua exposição ao conflito interparental a suas crenças sobre agressão. Simon e Furman ${ }^{32}$ informam que entre os meninos que fizeram avaliações negativas de conflito interparental, este previu um maior engajamento em conflitos e em agressões físicas. Em contraste, o conflito interparental foi associado com menor engajamento em conflitos e mais inícios de mudanças positivas entre as meninas.

Segundo esses autores, uma possível explicação para esses diferentes efeitos em meninos e meninas residiria nas diferenças de gênero construídas no processo de socialização, as quais orientariam as meninas para os relacionamentos e os meninos para um foco mais individual. Sugere-se, portanto, que meninas que testemunham conflitos parentais podem ser mais sensíveis ao potencial do conflito em causar danos ao relacionamento, enquanto os meninos poderiam se concentrar mais na funcionalidade da agressão em alcançar a dominância. Assim, os meninos que testemunham altos níveis de conflito podem interpretar a agressão como uma forma de alcançar objetivos em um relacionamento, enquanto as meninas podem perceber a agressão como algo que é prejudicial para os relacionamentos ${ }^{36}$. Simon e Furman ${ }^{32}$ destacam a tendência das meninas participantes do estudo em evitar conflitos no namoro, ou mesmo amenizá-los, dentre aquelas que avaliaram negativamente o conflito interparental.

Todavia, é importante ressaltar que tais estudos enfocaram a violência física ${ }^{32}$ ou o somatório de todas as violências ${ }^{36}$, o que pode não servir diretamente como modelo explicativo para a violência psicológica, que congrega manifestações explícitas e sutis. Desta forma, diversas manifestações de violência psicológica, muitas vezes, nem são consideradas agressão, passando despercebidas pelos adolescentes, que a praticam de forma corriqueira e frequente $e^{6,26,46}$, contribuindo assim para sua invisibilidade ${ }^{10}$.

Nosso estudo mostra que as meninas tendem a perpetrar mais violência psicológica no namoro que os meninos, a despeito da violência psicológica que vivenciam em outros contextos. Tal resultado é consistente com outras pesquisas em que as meninas relatam praticarem mais violência psicológica do que os meninos $2,3,6,19,46$. A percep- 
ção de que a violência psicológica é algo mais comum entre as mulheres é mostrada por Sears et al. ${ }^{3}$ e Oliveira et al. ${ }^{26}$, revelando mais uma vez a expressão das normas de gênero tradicionais em que meninas são encorajadas a falar sobre seus sentimentos e a expressar sua raiva verbalmente, algo interditado socialmente aos meninos, a quem restaria a expressão física da raiva e outros sentimentos negativos diante de um conflito.

Ao citar diversos estudos que associam testemunhar violência entre os pais àquela entre parceiros íntimos na vida adulta, Frieze ${ }^{2}$ afirma que essa relação pode ser especialmente forte nas mulheres.

Entretanto, os estudos sobre a diferença de impacto das violências perpetradas por pais e mães no namoro de seus filhos não são consensuais. No estudo de Kaura e Allen ${ }^{44}$ a perpetração feminina de violência no namoro está relacionada com a violência do pai, enquanto que a perpetração masculina de violência no namoro relaciona-se com a violência da mãe, ou seja, somente a violência sofrida pelos pais do sexo oposto foram preditores significantes da prática da violência no namoro nesse estudo. Jankowski et al. ${ }^{45}$, opostamente, afirmam que os entrevistados que presenciaram agressão física conjugal praticada pelo genitor do mesmo sexo estavam em maior risco de perpetrar o mesmo tipo de agressão no namoro, ao passo que aqueles que presenciaram apenas o genitor do sexo oposto agredir o parceiro não estavam em risco.

Foshee et al. ${ }^{47}$ por outro lado, reportam que a violência materna é mais significativa do que a paterna no que tange à influência na violência no namoro. Recentemente, Templea et al. ${ }^{48}$ afirmaram que para adolescentes do sexo feminino, houve uma associação entre a exposição à violência interparental (pai-mãe e mãe-pai) e perpetração de violência física e psicológica contra o parceiro; já entre os rapazes, houve apenas associação entre a violência da mãe contra o pai e a perpetração de violência no namoro. Além disso, para meninas e meninos, a relação entre a violência da mãe contra o pai e perpetração de violência no namoro foi totalmente mediada por atitudes de aceitação da violência. Como se pode perceber, para além das discordâncias, constata-se que níveis mais altos de violência parental experimentado durante a infância estariam associados com níveis mais elevados de perpetração de violência no namoro entre adolescentes.

Em relação à vivência de violência no grupo de amigos (entre pares) e sua relação com violência no namoro entre os adolescentes, nossos re- sultados corroboram outras pesquisas que mostram que fazer parte de grupos de colegas/amigos abusivos está positivamente relacionado com a prática de violência no namoro ${ }^{30,31,34,36}$.

Arriaga e Foshee ${ }^{31}$ afirmam que apenas a violência entre amigos prediz consistentemente a violência no namoro, em detrimento da associação entre violência interparental e a que ocorre no namoro dos adolescentes. Kinsfogel e Grych ${ }^{36}$ e Connolly e Goldberg ${ }^{30}$ discutindo sobre o papel do grupo de amigos na adolescência, afirmam que os mesmos são importantes influências nessa faixa etária, pois funcionam como modelos comportamentais e contribuem na construção de normas individuais e valores a respeito de interações sociais. Assim, a forma pela qual os amigos lidam com os conflitos em seus relacionamentos afetivo-sexuais pode ser tão ou mais importante do que a dos pais. Por ser o namoro um tema relevante e frequente em conversas entre amigos adolescentes ${ }^{30}$, a aprovação ou rejeição dos pares em relação às agressões cometidas entre namorados pode exercer uma forte influência sobre o comportamento do adolescente ${ }^{36}$, orientando a escolha de novos namorados e o curso desses relacionamentos ${ }^{34}$.

Já Levendosky et al. ${ }^{33}$ ao investigarem o papel de apoio social dos colegas na previsão de agressão no namoro, descobriram que os amigos podem influenciar as relações dos adolescentes no namoro para melhor ou para pior, incentivando ou rejeitando atitudes violentas. Assim, sugerese que um comportamento violento praticado ou endossado pelo grupo de amigos pode influenciar a mesma prática no namoro. Kinsfogel e $\mathrm{Grych}^{36}$, além de evidenciarem a importância do papel que as relações entre pares desempenham nas relações de namoro, também as consideram relevantes na compreensão de como o conflito interparental pode levar a agressão entre namorados. Mostram que jovens de lares mais agressivos associam-se mais com colegas com maior propensão a comportamentos abusivos, e estes grupos de pares podem desenvolver suas próprias "normas", apoiando ou até mesmo incentivando as agressões entre namorados.

No presente artigo, constata-se que o aumento do número de eventos de violência psicológica perpetrada por adolescentes também está relacionado com a maior frequência da vivência de violência psicológica entre irmãos e nos namoros anteriores.

Em revisão sobre a violência familiar e comportamento agressivo e transgressor na infância, Pesce ${ }^{49}$ afirma que a violência psicológica está 
presente em modelos coercitivos de educação, integrando, portanto, uma dinâmica de coerção dos filhos. Em artigo que aborda os conflitos severos entre irmãos como uma forma de violência familiar prejudicial ao desenvolvimento de crianças e adolescentes, a autora encontra a relação direta entre comportamentos destrutivos entre irmãos e o relato materno de comportamento transgressor de seus filhos (Garcia et al. apud Pesce $\left.{ }^{49}\right)$. Podemos reconhecer esse modelo coercitivo no estudo de Meneghel et al..$^{50} \mathrm{em}$ que a pedagogia da violência se concretiza em menos flexibilidade e diálogo por um lado, e por mais rigidez nas relações e nos papéis desempenhados por outro, gerando mais conflitos entre os irmãos. Embora o foco tenha sido as punições fisicamente violentas, pode-se afirmar que o estudo aponta para uma dinâmica de comunicação violenta na família que se evidencia também na relação entre irmãos, o que vemos em nossos resultados. Nesse sentido, as autoras destacam que os adolescentes punidos tiveram oito vezes mais chance de serem violentos com os irmãos.

A partir de relatos de meninas que sofreram violência intrafamiliar, De Antoni e Koller ${ }^{51}$ afirmam que há uma relação de poder dos irmãos mais velhos sobre os mais novos, o que poderia desencadear brigas e coerções, muitas vezes demarcando uma posição hierarquicamente superior do irmão mais velho, que agrediria quando repudiado em seu comando, ou coagindo os que obedecem.

Sobre a recorrência de violência psicológica em relacionamentos afetivo-sexuais, Ramire ${ }^{52}$ et al. e Bonomi et al. ${ }^{53}$ destacam que em contraste com estudos com população adulta, cuja maioria de mulheres e homens indicaram apenas um parceiro abusivo, os adolescentes tenderam a relatar que se relacionaram com mais de um parceiro que havia perpetrado violência no namoro contra eles.

Nesse estudo, entre as mulheres que relataram violência no namoro, mais de um terço relatou ter vivenciado violência de dois ou mais parceiros, dentre os quais se destacam expressões de violência psicológica: o comportamento controlador (35,6\%); humilhações/xingamentos $(37,0 \%)$; insultos $(44,3 \%)$; e ameaças $(62,5 \%)$. Uma proporção considerável de homens que sofreram violência no namoro também disseram que tiveram dois ou mais parceiros que perpetraram violência, como se segue: o comportamento de controle $(42,1 \%)$; insultos $(51,2 \%)$; baixos postos $(53,3 \%)$, ameaças $(55,6 \%)$ e chamadas indesejadas/mensagens de texto $(60,7 \%)$.
Homens e mulheres raramente relataram um incidente isolado de violência no namoro.

No que se refere ao aspecto etário, reitera-se que a violência entre parceiros íntimos na adolescência se processa de maneira diversa do que nos adultos ${ }^{54}$, constatando-se, entre os primeiros, a simetria entre os gêneros no que se refere a perpetrar e sofrer violências no namoro ${ }^{6,19,26,43,55-}$ 57. Assim, destacamos que os achados em estudos com adultos não devem ser transpostos diretamente para amostras com adolescentes, sobretudo por estes estarem em fase de experimentações em que as manifestações de violência nas relações íntimas ainda são mais similares entre os gêneros, ao passo que entre adultos, normas de gênero estão mais recrudescidas, tendendo as manifestações da violência a ocorrer mais próximas dos padrões tradicionais que localizam a violência na masculinidade $e^{58,59}$.

Ressalta-se que o presente artigo não apresenta relação de causalidade entre vivências anteriores de violência psicológica e aquela que ocorre no namoro do adolescente, pois a metodologia empregada na pesquisa não permite tal afirmação. Os resultados obtidos apontam para a concomitância das formas de violência psicológica ao longo do desenvolvimento e para o aumento do número de eventos desse tipo de violência, perpetrada por adolescentes, relacionado à vivência de violências psicológicas em outros contextos relacionais.

A teoria da Aprendizagem Social ${ }^{60}$ trata da transmissão intergeracional da violência, em que comportamentos seriam aprendidos por imitação, podendo ser reproduzidos vida afora. De acordo com esta teoria, ser vítima de maus-tratos ou testemunha de agressões frequentes na família e em outros contextos de convivência influenciaria a percepção, podendo legitimar diferentes tipos de violência como formas de resolver conflitos. Kim et al. ${ }^{61}$ apontam outra forma de compreensão, afirmando que conflitos de relacionamento em uma geração não predizem diretamente outros na próxima, discordando de que a transmissão intergeracional de conflito de relacionamento ocorra através da aprendizagem direta. Estes autores apostam na desregulação emocional da família e em pouca disciplina por parte dos pais como preditores do resultado de conflito interparental. Isso apoiaria o argumento de que a capacidade do indivíduo de regular suas emoções e os comportamentos emocionalmente relacionados (por exemplo, raiva, hostilidade) desempenha um papel significativo nas associações de conflito de relacionamento trans- 
geracionais. Assim, para estes autores, a desregulação emocional seria um importante mecanismo subjacente ao moldar relacionamentos românticos em gerações. A partir desses posicionamentos, percebe-se a necessidade de avanço do conhecimento no que se refere ao aprendizado da violência e da complexidade dos contextos onde se dão os relacionamentos afetivos-sexuais dos adolescentes na sociedade atual.

\section{Colaboradores}

QBM Oliveira trabalhou na concepção teórica, elaboração e redação final do texto; SG Assis participou da concepção teórica e discussão dos resultados; K Njaine participou da redação final; T Pires realizou a organização e a análise dos dados. 


\section{Referências}

1. Cornelius TL, Sullivan KT, Wyngarden N, Milliken JC. Participation in prevention programs for dating violence: beliefs about relationship violence and intention to participate. J Interpers Violence 2009; 24(6):1057-1078.

2. Frieze I. Female violence against intimate partners: an introduction. Hanson Psychology of Women Quarterly 2005; 29(3):229-237.

3. Sears HA, Byers ES, Whelan JJ, Saint-Pierre M. If it hurts you, then it is not a joke: adolescents' ideas about girls and boys use of abusive behavior in dating relationships. J Interpers Violence 2006; 21(9):1191-1207.

4. Oliveira QBM, Assis SG, Njaine K, Pires TO. Gênero e violência física no namoro de adolescentes de dez capitais brasileiras. Psicologia, Teoria e Pesquisa-UNB. No prelo 2013.

5. Minayo MCS, Assis SG, Njaine K, organizadores. Amor e violência: um paradoxo de namoro e do 'ficar' entre jovens brasileiros. Rio de Janeiro: Fiocruz; 2001.

6. Fernández-Fuertes AA, Fuertes A. Physical and psychological aggression in dating relationships of spanish adolescents: motives and consequence. Child Abuse Negl 2010; 34(3):183-191.

7. Cascardi M, Avery-Leaf S, O'Leary KD, Slep AMS. Factor structure and convergent validity of the Conflict Tactic Scale in high-school students. Psychol Assess 1999; 11(4):546-555.

8. Jackson SM. Teenage dating violence [thesis]. New Zealand: University of Auckland; 1998.

9. Jezl D, Molidor C, Wright T. Physical, sexual and psychological abuse in high school dating relationships: prevalence rates and self esteem issues. Child Adolesc Social Work J 1996; 13(1):69-87.

10. Abranches CD, Assis SG. A (in)visibilidade da violência psicológica na infância e adolescência no contexto familiar. Cad Saúde Publica 2011; 27(5):843854

11. O'Keefe NK, Brockopp K, Chew E. Teen dating violence. Soc Work 1986; 31(6):463-468.

12. Makepeace JM. Courtship violence among college students. Fam Relat 1981; 30(1):97-102.

13. Makepeace JM. Gender differences in courtship violence victimization. Fam Relat 1986; 35(3):383388.

14. Aizenman M, Kelley G. The incidence of violence and acquaintance rape in dating violence relationships among college men and women. J Coll Stud Dev 1988; 29(4):305-311.

15. Saavedra R, Machado C, Martins C, Vieira D. Inventário de conflitos nas relações de namoro entre adolescentes (ICRNA). In: Simões MR, Machado $\mathrm{C}$, Gonçalves $\mathrm{M}$, coordenadores. Instrumentos e contextos de avaliação psicológica. Coimbra: Almedina; 2011.

16. Rodríguez Francos L, Antuña Bellerín ML, LópezCepero Borrego J, Rodríguez Díaz FJ, Bringas Molleda C. Tolerance towards dating violence in spanish adolescents. Psicothema 2012; 24(2):236-242.

17. Shen AC, Chiu MY, Gao J. Predictors of dating violence among chinese adolescents: the role of gender-role beliefs and justification of violence. $J$ Interpers Violence 2012; 27(6):1066-1089.
18. Hamby S, Nix K, De Puy J, Monnier S. Adapting dating violence prevention to francophone Switzerland: a story of intra-western cultural differences. Violence Vict 2012; 27(1):33-42.

19. Schiff M, Zeira A. Dating aggression and sexual risk behaviours in a sample of at-risk Israeli Youth. Child Abuse Negl 2005; 29(11):1249-1263.

20. Rivera-Rivera L, Allen B, Rodríguez-Ortega G, Chávez-Ayala $R$, Lazcano-Ponce E. Violencia durante el noviazgo, depresión y conductas de riesgo en estudiantes femeninas (12-24 años). Salud Publica Mex 2006; 48(Supl. 2):S288-S296.

21. Antonio T, Hokoda A. Gender Variations in Dating Violence and Positive Conflict Resolution Among Mexican Adolescents. Violence Vict 2009; 14(4):533545.

22. Gomez AM, Speizer IS, Moracco KE. Linkages between gender equity and intimate partner violence among urban brazilian youth. J Adolesc Health 2011; 49(4):393-399.

23. Garbarino J, Guttamann E, Seeley JW. The psychologically battery child. San Francisco: Jossey-Bass Publishers; 1986.

24. O'Leary KD, Slep AM. A dyadic longitudinal model of adolescent dating aggression. J Clin Child Adolesc Psychol 2003; 32(3):314-327.

25. Molidor CE. Gender differences of psychological abuse in high school dating relationships. Child Adolesc Social Work J 1995; 12(2):119-134.

26. Oliveira QBM, Assis SG, Njaine K, Oliveira RVC. Violências nas relações afetivo-sexuais. In: Minayo MSC, Assis SG, Njaine K, organizadores. Amor e violência: um paradoxo das relações de namoro e do 'ficar' entre jovens brasileiros. Rio de Janeiro: Fiocruz; 2011.

27. Wolfe DA, Scott K, Reitzel-Jaffe D, Wekerle C. Development and validation of the Conflict in Adolescent Dating Relationships Inventory. Psychol Assess 2001; 13(2):277-293.

28. Fernet M. Amour, violence et adolescence. Quebéc: Presses de d'Université du Québec; 2005.

29. O'Keefe M. Predictors of dating violence among high school students. J Interpers Violence 1997; 12(4):546-568.

30. Connolly JA, Goldberg A. Romantic relationships in adolescence: the role of friends and peers in their emergence and development. In: Furman W, Brown BB, Feiring C, editores. The development of romantic relationships in adolescence. New York: Cambridge University Press; 1999. p. 266-290.

31. Arriaga XB, Foshee VA. Adolescent dating violence: do adolescents follow in their friends', or their parents', footsteps? J Interpers Violence 2004; 19(2):162184.

32. Simon VA, Furman W. Interparental conflict and adolescents' romantic relationship conflict. $J$ Res Adolescence 2010; 20(1):188-209.

33. Levendosky AA, Huth-Bocks A, Semel MA. Adolescent peer relationships and mental health functioning in families with domestic violence. J Clin Child Adolesc Psychol 2002; 31:206-218. 
34. Harper GW, Gannon C, Watson SE, Catania JA, Dolcini MM. The role of close friends in african american adolescents' dating and sexual behavior. I Sex Res 2004; 41(4):351-362.

35. Ozer E, Tschann L, Flores E. Violence perpetration across peer and partner relationships: co-occurrence and longitudinal patterns among adolescents. J Adolesc Health 2004; 34(1):64-71.

36. Kinsfogel KM, Grych JH. Interparental conflict and adolescent dating relationships: Integrating cognitive, emotional, and peer influences. J Family Psychol 2004; 18(3):505-515.

37. Straus MA. Measuring intra family conflict and violence: the Conflict Tactics Scale. J Marriage Fam 1979; 41(1):75-88.

38. Hasselmann MH, Reichenheim ME. Adaptação transcultural da versão em português das "Conflict Tactics Scales Form R" (CTS-1) usada para aferir violência no casal: equivalências semântica e de mensuração. Cad Saude Publica 2003; 19(4):1083-1093.

39. Fumes G, Corrente JE. Modelos inflacionados de zeros: aplicações na análise de um questionário de frequência alimentar. Rev Bras Biom 2010; 28(1):2438.

40. Lambert D. Zero-inflated poisson regression with an application to defects in manufacturing. Technometrics 1992; 34(1):1-14.

41. O'Keefe M, Treister L. Victims of dating violence among high school students: are the predictors different for males and females? Violence Against Women 1998; 4(2):195-223.

42. Schwartz M, O'Leary SG, Kendziora KT. Dating aggression among high school students. Violence Vict 1997; 12(4):295-305.

43. Wolfe DA, Scott K, Wekerle C, Pittman A. Child maltreatment: risk of adjustment problems and dating violence in adolescence. J Am Acad Child Adolesc Psychiatry 2001; 40(3):282-298.

44. Kaura SA, Allen CM. Dissatisfaction with relationship power and dating violence perpetration by men and women. J Interpers Violence 2004; 19(5): 576-588.

45. Jankowski MK, Leitenberg H, Henning K, Coffey P. Intergenerational transmission of dating aggression as a function of witnessing only same sex parents vs. opposite sex parents vs. parents as perpetrators of domestic violence. J Fam Violence 1999; 14(3): 267-279.

46. Sears HA, Byers ES, Price EL. The co-occurrence of adolescent boys' and girls' use of psychologically, physically, and sexually abusive behaviours in their dating relationships. J Adolesc 2007; 30(3):487-504.

47. Foshee V, Bauman K, Linder F. Family violence and the perpetration of adolescent dating violence: Examining social learning and social control processes. J Marriage Fam 1999; 61(2):331-342.

48. Templea JR, Shoreyb RC, Tortoleroc SR, Wolfe DA, Gregory L. Stuartb GL. Importance of gender and attitudes about violence in the relationship between exposure to interparental violence and the perpetration of teen dating violence. Child Abuse Negl 2013; 37(5):343-352.
49. Pesce RP. Problemas de comportamento externalizantes na infância: a violência em foco [tese]. Rio de Janeiro: Escola Nacional de Saúde Pública Sergio Arouca, Fundação Oswaldo Cruz; 2009.

50. Meneghel SN, Giuliani EJ, Falceto O. Relações entre violência doméstica e agressividade na adolescência. Cad Saude Publica 1998; 14(2):327-335.

51. De Antoni C, Koller SH. A visão de família entre adolescentes que sofreram violência intrafamiliar. Estudos de Psicologia 2000; 5(2):347-381.

52. Ramirez M, Paik A, Sanchagrin K, Heimer K. Violent peers, network centrality, and intimate partner violence perpetration by young men. $J$ Adolesc Health 2012; 51(5):503-509.

53. Bonomi AE, Anderson ML, Nemeth J, Bartle-Haring S, Buettner C, Schipper D. Dating violence victimization across the teen years: abuse frequency, number of abusive partners, and age at first occurrence. BMC Public Health 2012; 10(12):637.

54. Wekerle C, Wolfe DA. The role of child maltreatment and attachment style in adolescent relationship violence. Dev Psychopathol 1998; 10(3):571-586.

55. Malik S, Sorenson SB, Aneshensel CS. Community and dating violence among adolescents: perpetration and victimization. J Adolesc Health 1997; 21(5): 291-302.

56. Molidor C, Tolman RM. Gender and contextual factors in adolescent dating violence. Violence Against Women 1998; 4(2):180-194.

57. Antônio T, Hokoda A. Gender variations in dating violence and positive conflict resolution among mexican adolescents. Violence Vict 2009; 24(4):533-545.

58. Saffioti HIB. Gênero, patriarcado e violência. São Paulo: Fundação Perseu Abramo; 2004.

59. Bourdieu P. A dominação masculina. Rio de Janeiro: Bertrand Brasil; 2011.

60. Bandura A. Aggression: a social learning analysis. Englewood Cliffs: Prentice-Hall; 1973.

61. Kim HK, Pears KC, Capaldi DM, Owen D. Emotion dysregulation in the intergenerational transmission of romantic relationship conflict. J Fam Psychol 2009; 23(4):585-595.

Artigo apresentado em 05/09/2013

Aprovado em 04/10/2013

Versão final apresentada em 07/10/2013 